\title{
SEPTEMBRE-OCTOBRE 1986
}

\section{8* ANNÉE - N 761}

\section{revue internationale de la croix-rouge}

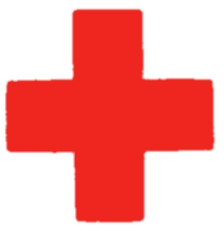

INTER ARMA CARITAS

\section{GENËVE}

COMITÉ INTERNATIONAL DE LA CROIX-ROUGE FONDÉ EN 1863 
M. ALEXANDRE HAY, Docteur honoris causa des Universités de Genève et de Saint-Gall, avocat, ancien directeur général de la Banque nationale suisse, président (membre depuis 1975)

$\mathbf{M}^{\text {me }}$ DENISE BINDSCHEDLER-ROBERT, docteur en droit, ancien professeur à l'Institut universitaire de hautes études internationales, Genève, juge à la Cour européenne des droits de l'homme, vice-présidente (1967)

MM. MAURICE AUBERT, docteur en droit, vice-président (1979)

JACQUES F. DE ROUGEMONT, docteur en médecine (1967)

ULRICH MIDDENDORP, docteur en médecine, chef de la clinique chirurgicale de l'Hôpital cantonal, Winterthour (1973)

HENRY HUGUENIN, banquier (1974)

RICHARD PESTALOZZI, docteur en droit, ancien vice-président du CICR (1977)

ATHOS GALLINO, docteur en médecine, maire de Bellinzone (1977)

ROBERT KOHLER, docteur ès sciences économiques (1977)

RUDOLF JÄCKLI, docteur ès sciences (1979)

DIETRICH SCHINDLER, docteur en droit, professeur à l'Université de Zurich (1961$1973 ; 1980)$

HANS HAUG, docteur en droit, ancien professeur à l'Ecole des hautes études économiques et sociales de Saint-Gall, ancien président de la Croix-Rouge suisse (1983)

PETER ARBENZ, licencié ès sciences politiques (1983), (en congé depuis mars 1986)

PIERRE KELLER, docteur en philosophie en relations internationales (Yale), banquier (1984)

RAYMOND R. PROBST, docteur en droit, ancien ambassadeur de Suisse, ancien secrétaire d'Etat au Département fédéral des Affaires étrangères à Berne (1984)

ODILO GUNTERN, docteur en droit, ancien député au Conseil des Etats (1985)

ANDRÉ GHELFI, ancien secrétaire central et vice-président de la Fédération suisse des travailleurs de la métallurgie (1985)

$M^{\text {me }}$ RENÉE GUISAN, secrétaire générale de l'«Institut de la Vie» international, membre de la fondation suisse Pro Senectute, membre de l'«International Association for Volunteer Effort" (1986)

MM. DANIEL FREI, professeur de science politique à l'Université de Zurich (1986)

ALAIN B. ROSSIER, docteur en médecine, ancien professeur de rééducation des paraplégiques à l'Université de Harvard, privat-docent à la faculté de médecine de I'Université de Genève, président de la Société médicale internationale de paraplégie (1986)

\title{
CONSEIL EXÉCUTIF
}

\author{
M. ALEXANDRE HAY, président \\ M. MAURICE AUBERT \\ M. RICHARD PESTALOZZI \\ M. ATHOS GALLINO \\ M. RUDOLF JÄCKLI \\ M. PIERRE KELLER \\ M. ANDRÉ GHELFI
}

Le Comité international de la Croix-Rouge (CICR) est, avec la Ligue des Sociétés de la Croix-Rouge et du Croissant-Rouge et les 139 Sociétés nationales de la Croix-Rouge et du Croissant-Rouge reconnues, l'une des trois composantes de la Croix-Rouge internationale.

Institution humanitaire indépendante, le CICR est l'organe fondateur de la Croix-Rouge. Intermédiaire neutre en cas de conflits armés et de troubles, il s'efforce d'assurer, de sa propre initiative ou en se fondant sur les Conventions de Genève, protection et assistance aux victimes des guerres internationales et civiles, des troubles et tensions internes, apportant ainsi sa contribution à la paix dans le monde. 


\section{REVUE INTERNATIONALE \\ DE LA CROIX-ROUGE}

ISSN 0035-3361

SOMMAIRE

COMITÉ INTERNATIONAL DE LA CROIX-ROUGE

DANS LE MONDE DE LA
CROIX-ROUGE

FAITS ET DOCUMENTS

LIVRES ET REVUES
SEPTEMBRE-OCTOBRE $1986-N^{\circ} 761$

Vangah Francis Wodie: L'Afrique et le droit humanitaire

Jean de Preux: Texte de synthèse VI: Secours

Commentaire des Protocoles additionnels du 8 juin 1977 aux Conventions de Genève du 12 août 1949 . . . . . . . . . . . . .

Reconnaissance de la Société de la CroixRouge de Guinée-Bissau . . . . . . . . . . 290

Reconnaissance de la Société du CroissantRouge des Emirats Arabes Unis . . . . . . 292

Activités extérieures:

Afrique - Amérique latine - Asie - Moyen-

Orient . . . . . . . . . . . . . . . 294

L'été de la diffusion . . . . . . . . . . 301

Un an après la catastrophe de Mexico . . . 304

Résolution de l'Organisation de l'Unité Africaine relative au CICR ... . . . . . . 306

Huitième Conférence des chefs d'Etat ou de Gouvernement des pays non alignés . . . 308 Congrès international «La Paix et les actions humanitaires $» \ldots \ldots . \ldots . \ldots 308$

Völkerrecht im Dienste des Menschen Festschrift für Hans Haug . . . . . . . . 313

Charité-Business (Bernard Kouchner) . . . . 314

Droit humanitaire et conflits internes (Rosemary Abi-Saab) ........... 317

Mouvements de réfugiés .......... 320

Annuaire 1985 de l'Institut international de droit humanitaire ........... 322

Dans les Revues ............. 323 
La Revue internationale de la Croix-Rouge est publiée par le Comité international de la Croix-Rouge (CICR) depuis 1869. Elle a porté à l'origine le titre de «Bulletin international des Sociétés de secours aux militaires blessés», puis de "Bulletin international des Sociétés de la Croix-Rouge».

- Organe officiel du CICR et de la Croix-Rouge internationale,

- publication spécialisée dans le droit international humanitaire et la doctrine du CICR,

- chronique des activités internationales de la Croix-Rouge, ouvrage de référence essentiellement, qui garde la mémoire des événements,

la Revue internationale maintient un courant d'informations et constitue le lien nécessaire entre les membres de la Croix-Rouge internationale.

La Revue internationale de la Croix-Rouge paraît, une fois tous les deux mois, en trois éditions principales:

en français: Revue INTERnationale de la Croix-Rouge

en anglais: International Review of tHe Red Cross (depuis 1961)

en espagnol: Revista Internacional de la Cruz Roja (depuis 1976)

Elle publie également, en allemand, un cahier d'Extraits des textes parus dans les éditions principales.

RÉDACTION: Jacques Meurant, rédacteur en chef

ADResSE: $\quad$ Revue internationale de la Croix-Rouge

17, avenue de la Paix

$\mathrm{CH}$ - 1202 - Genève, Suisse

ABONNEMENTs: un an 30 francs suisses; le numéro 5 francs.

Extraits en allemand: un an 10 francs; le numéro 2 francs.

Compte de chèques postaux: 12 - 1767 Genève

Compte bancaire: 129.986 Société de Banque Suisse, Genève

Seuls les textes signés par le Comité international de la Croix-Rouge engagent la responsabilité de celui-ci. 